Thorax (1969), 24, 91.

\title{
Pleuro-pulmonary amoebiasis
}

\author{
B. T. LE ROUX \\ From the Thoracic Surgical Unit, Wentworth Hospital, University of Natal
}

\begin{abstract}
Experience with amoebic infestation of the pleural space and the lung in an area where amoebiasis is endemic is described. The manifestations of pleuro-pulmonary amoebiasis are outlined, and, for completeness, a note is added on pericardial amoebiasis and the technique of pericardiocentesis.
\end{abstract}

From a retrospective scrutiny of 500 consecutive case records, which related to patients with empyema thoracis, over a 13-year period in a relatively closed community of one and a half million in the south-east region of Scotland (le Roux, 1965), it emerged that, in $50 \%$ of patients, empyema was related to non-specific pulmonary infection for which a persisting cause was not found, and that this was the commonest single cause for empyema in this region. Trauma was a rare cause, and amoebic empyema was not encountered. On completion of this survey the opportunity arose to establish a thoracic surgical unit which would serve the Province of Natal in South Africa. The population of this Province is some three million, of whom two million are Africans, half a million are Asiatics, and half a million are of Caucasian stock. Empyema thoracis in the first 200 examples of this disease $(80 \%$ of which were in relatively young men) seen in this new community was a late and probably iatrogenic complication of traumatic haemothorax in $50 \%$, and in nearly all these patients the trauma was a stab wound. In the remainder the commonest cause of empyema was amoebiasis and in most of these patients the pleural infection was an extension from the liver. The purpose of this paper is to relate the natural history of amoebiasis which has extended into the pleural space or the lung.

Extension of amoebiasis from the liver to the lungs and pleura is well documented, and the problems which relate to amoebiasis in clinical practice have been described by Wilmot (1962). Pleuro-pulmonary amoebiasis may develop as a consequence of :

(1) Direct extension (either abruptly by rupture of a liver abscess, or insidiously) from the liver through the diaphragm either $(a)$ to involve the pleura, here promoting the symptoms and signs of pleurisy, or with the development of a serous effusion or an empyema; or (b) into the lung with the development of pulmonary consolidation, lung abscess or broncho-hepatic fistula.

(2) Haematogenous dissemination to the lungs from the bowel or liver, with either single or multiple pulmonary abscesses, or localized or diffuse areas of pulmonary consolidation.

With regard to direct extension from the liver into the pleural space or lung, it is common to hear a pleural rub in a patient with an amoebic liver abscess. Such a rub may disappear with specific anti-amoebic treatment, or may be a prelude to the development of a serous pleural effusion or an amoebic empyema, the consequence of rupture of an amoebic abscess of the liver or sub-phrenic space into the pleural space. Rub and serous effusion are not inevitable precursors to the development of an empyema. Amoebic empyema thoracis may also result from direct extension of an amoebic lung abscess to the pleura. The development of amoebic empyema may be abrupt or insidious ; an insidious onset is more usual, but sometimes the onset is acute, with severe pain and shock, followed quickly by death. The right pleural cavity is more often involved (Figs 1 and 2) but occasionally a left-sided amoebic empyema is found (Figs 3, 4, 5, and 6).

There are well-documented cases of left amoebic empyema which has developed as a complication of amoebic pericarditis, itself the manifestation of direct extension of hepatic amoebiasis through the diaphragm into the pericardium. Pus obtained at thoracentesis in a patient with an amoebic empyema is usually 'anchovy' in colour but may be yellow or green; it must be examined both for pathogenic bacteria and, by appropriate techniques, for amoebae. Drug therapy for amoebiasis, in conjunction with intermittent closed drainage, is more often successful in amoebic empyema 
FIG. 1

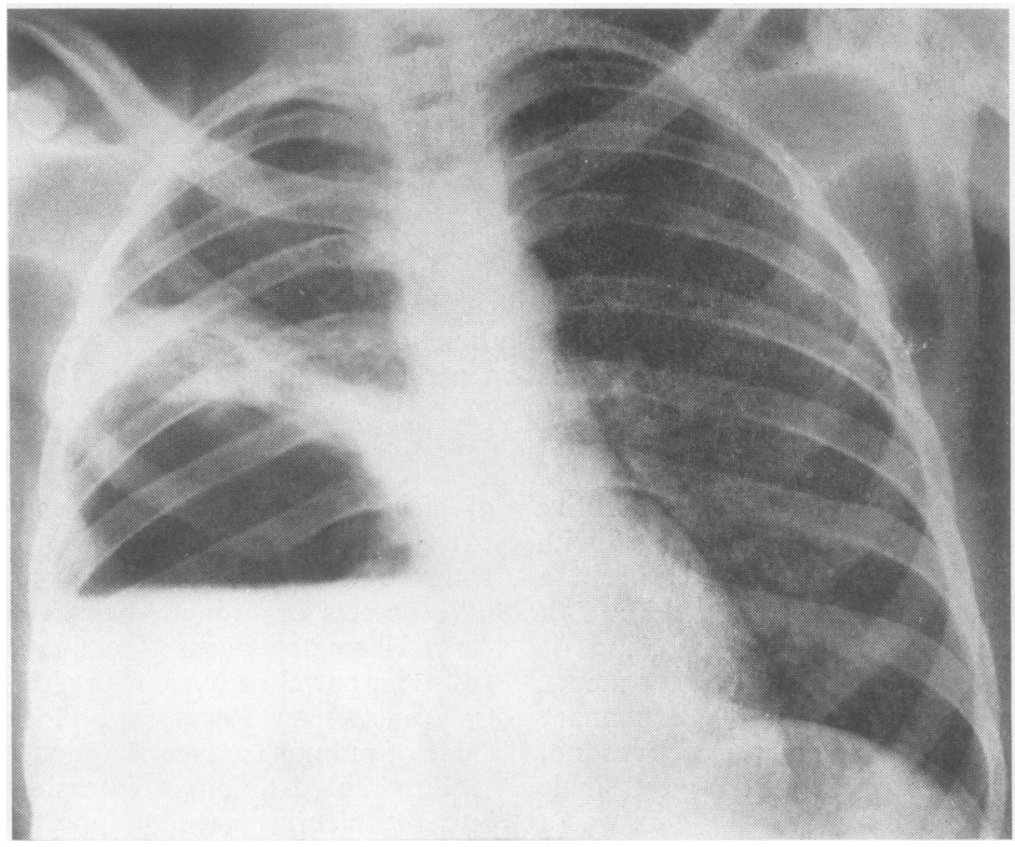

FIGS 1 and 2. Postero-anterior and lateral views show a large right empyema in which there is a level. Pus shown to contain Entamoeba histolytica was aspirated from the right pleura. Management was by continuous closed drainage and antiamoebic drugs. Convalescence was uneventful and the empyema rapidly diminished in size. Other surgical action was not required. When the empyema was greatly reduced in size, bile discharged into the water-seal for a few days. The biliary fistula had closed before the intercostal tube was removed.

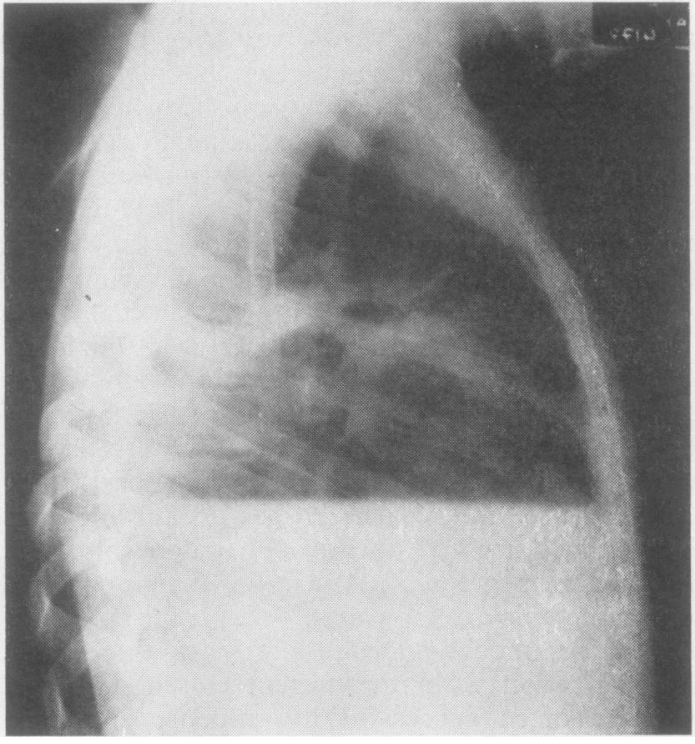

FIG. 2 thoracis than in other forms of empyema ; continuous closed drainage may be necessary, and occasionally open drainage, sometimes with drainage also of the sub-phrenic lesion.

Of 200 patients with empyema thoracis managed over a period of two years, 37 were established as amoebic. All the patients were of African or Asiatic stock; they represent only a small proportion of the total number of patients with amoebic empyema seen in the Province, and include those $\mathcal{O}$ in whom standard medical management (by appro- $N$ priate drugs and intermittent closed drainage by $N$ aspiration) had failed, or in whom the diagnosis $\omega$ of amoebiasis had not been entertained. Of the 37 patients, management by continuous closed $\stackrel{0}{=}$ drainage in conjunction with the exhibition of $\stackrel{\mathscr{\bigotimes}}{+}$ emetine and chloroquine in 30 resulted in rapid: clinical improvement and only slightly less rapid restoration of the radiographic appearances to nearly normal. Of the remaining seven patients, $\frac{\stackrel{\rho}{\mathbb{D}}}{Q}$ two were admitted moribund and died despite $\unrhd$ closed drainage and the exhibition of appropriate drugs. In two patients the amoebic empyema was $ᄋ$ 


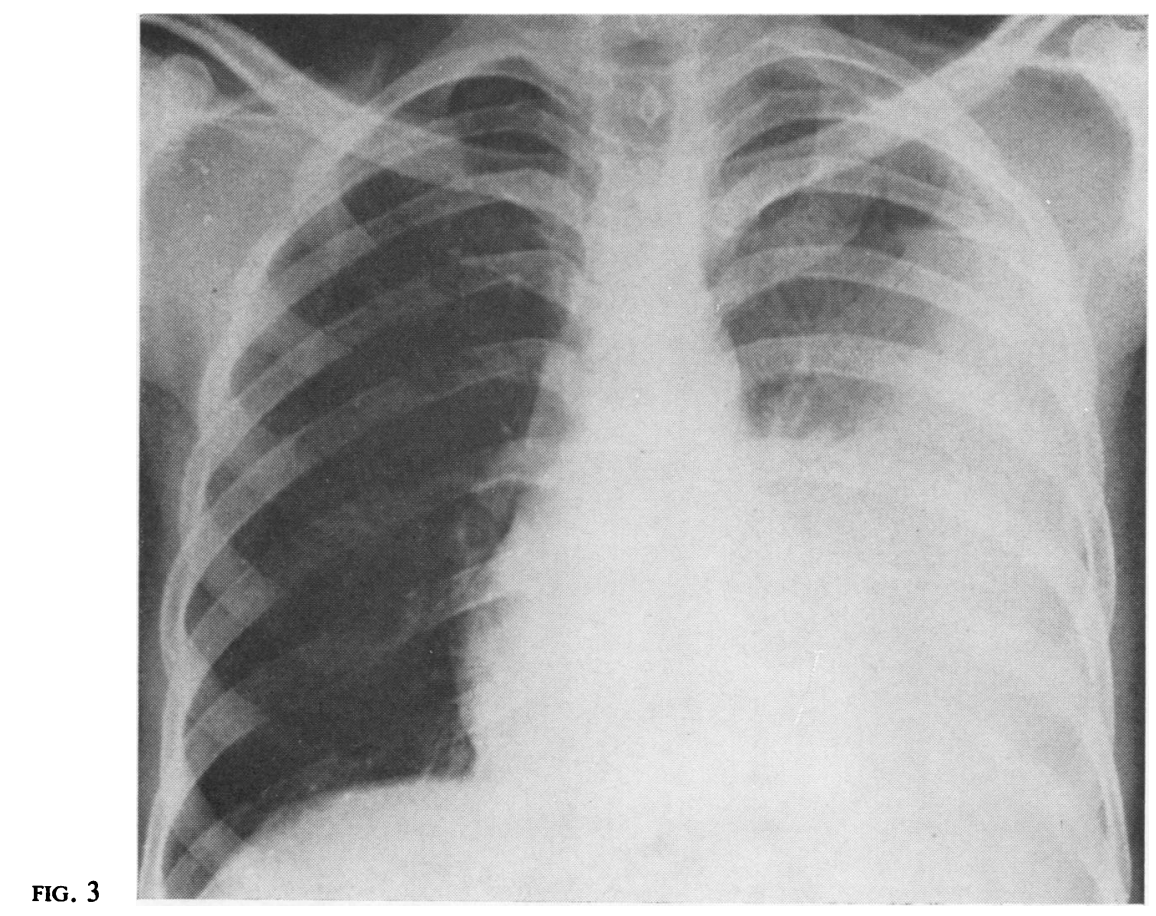

FIGS 3 and 4. The left pleural lesion shown in the postero-anterior and lateral films was ultimately established as an amoebic empyema and management was by closed drainage with appropriate drug therapy.

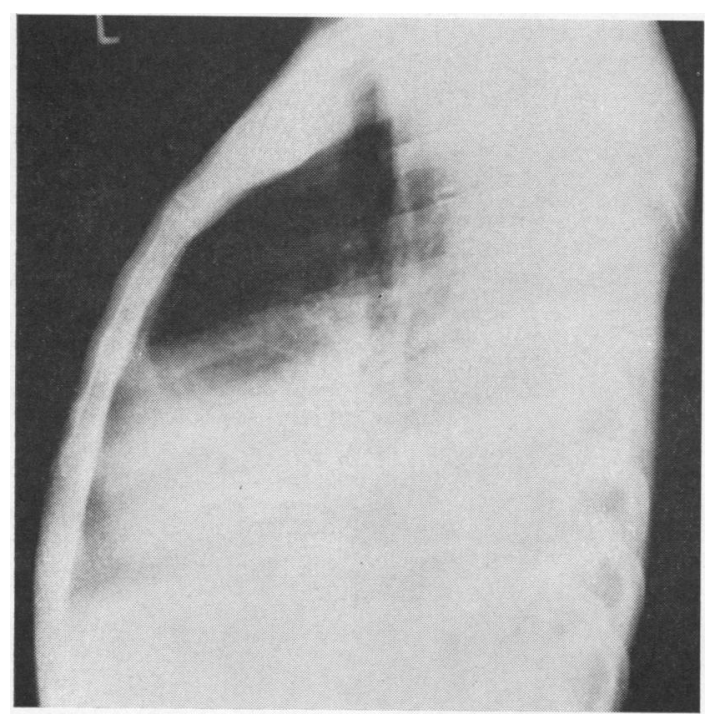

FIG. 4 secondarily infected with $E$. coli and management was first by closed and then by open drainage. In three patients secondary infection was with a variety of organisms, probably of iatrogenic origin, and management by closed drainage was followed by decortication. In one of these patients the amoebic empyema was left-sided and at decortication the empyema space was seen to communicate directly with the pericardial space, itself the site of amoebic infection, and there was communication between the pericardial space and the left lobe of the liver. Thirty-two of the 37 amoebic empyemata were right-sided, four were left-sided, and one was bilateral. In three of the four leftsided empyemata, amoebic pericarditis was a clinically recognized feature of the disease or was demonstrated at thoracotomy. In the patient with bilateral amoebic empyemata and in five of the patients with right-sided amoebic empyema there was unequivocal evidence (by pericardiocentesis) of amoebic pericarditis. In five patients it was necessary to manage hepatic amoebiasis by aspiration ; 


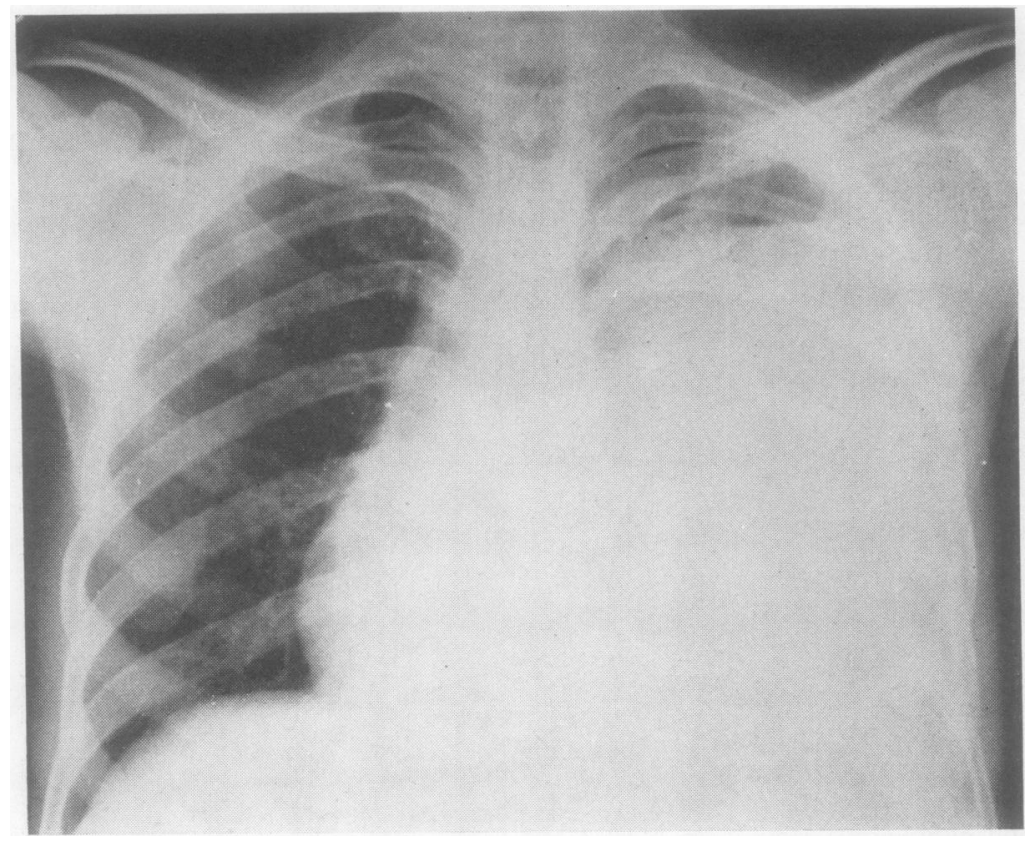

FIG. 5. Left amoebic empyema with amoebic pericarditis. Management was by closed drainage of the left pleural space and aspiration of the pericardium.

FIG. 6. This patient was known to have had amoebiasis and the left empyema was established as amoebic. The pleural shadow persisted despite closed drainage, and ultimately Escherichia 옹 coli was grown from the pus. Decortication was undertaken, at $\frac{\mathrm{O}}{\mathrm{C}}$ which it was established that there was a communication $N$ between the pericardial sac and the left pleura, that there was an 0 extensive amoebic pericarditis, $\omega$ and that the pericardium com- 0 municated directly with the left lobe of the liver, in which there was an incompletely closed amoebic abscess cavity. 


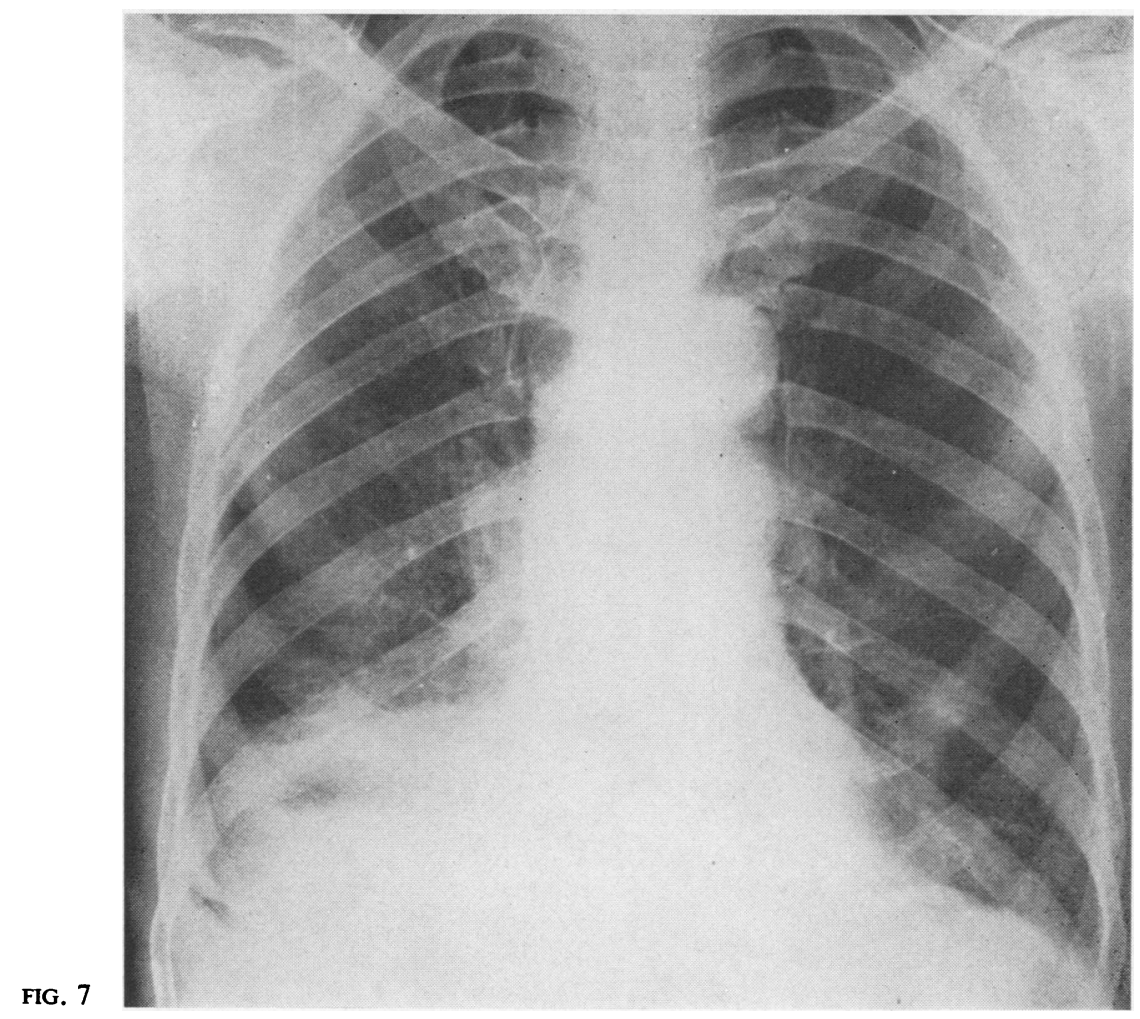

For legends to Figs 7 to 10 see p. 96 (overleaf).

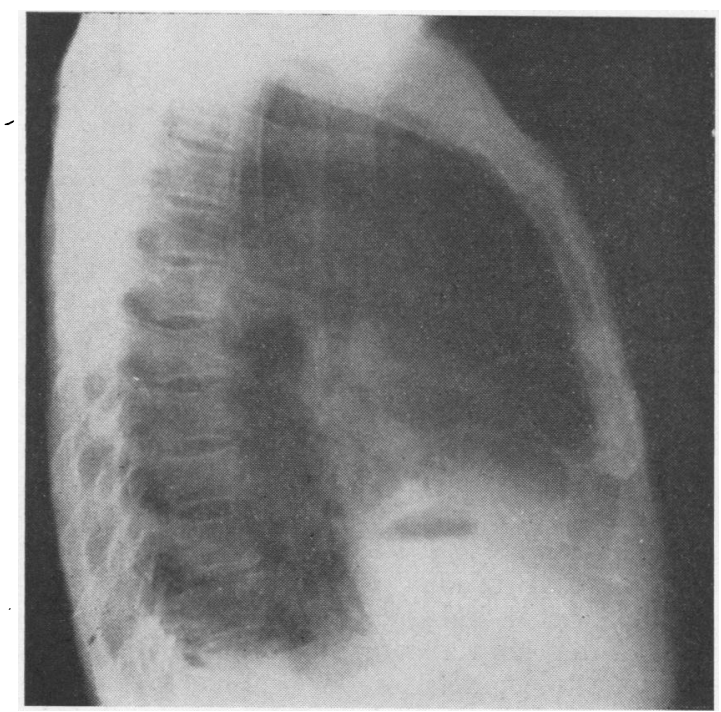

FIG. 8 in none was it necessary to manage an hepatic collection of amoebic pus by open drainage, and in only one was a subphrenic collection of pus drained surgically (Figs 7, 8, 9, and 10).

Trans-diaphragmatic migration of amoebae from the liver to the lung in lymphatic channels, without macroscopic evidence of rupture through the diaphragm, probably occurs. Pulmonary lesions and cavitation are most common in either the middle (Figs 11 and 12) or the right lower lobe (Figs 13, 14, 15, and 16), sometimes with abscess formation (Figs 17 and 18). The left lower lobe or the lingular segment of the left upper lobe may be the site of amoebic lung abscess; pulmonary involvement is occasionally bilateral. The usual manner of extension is by perforation of a subphrenic or hepatic abscess through the diaphragm, across an obliterated pleural space, with pulmonary consolidation or abscess; or, more commonly, direct extension to a bronchus with little involvement of the pulmonary parenchyma and evacuation of the subdiaphragmatic lesion by 


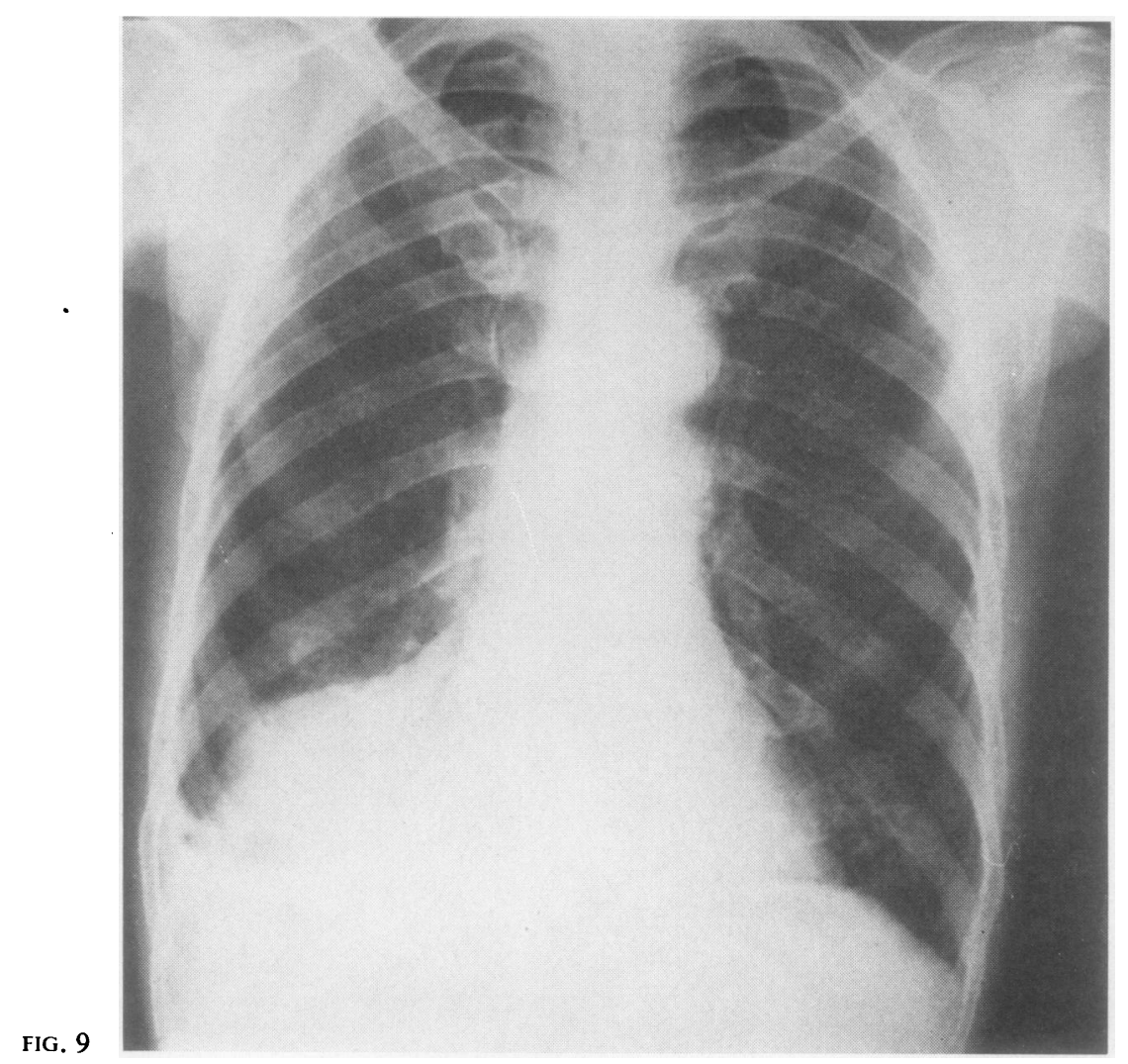

FIGS 7, 8, 9, and 10. Radiographs of the same patient made at intervals of one week. Presentation was with cough and purulent sputum. The right lower opacity in Figs 7 and 8 contains a level. The gel diffusion test was positive. The patient was presumed to have an amoebic lung abscess. In Figs 9 and 10 the level has disappeared. Response to anti-amoebic drugs was unimpressive clinically and radiographically, and the possibility of another cause for the 'pulmonary' lesion was considered an indication for exploratory thoracotomy. The lesion was shown to be a subphrenic abscess in which there were amoebae.

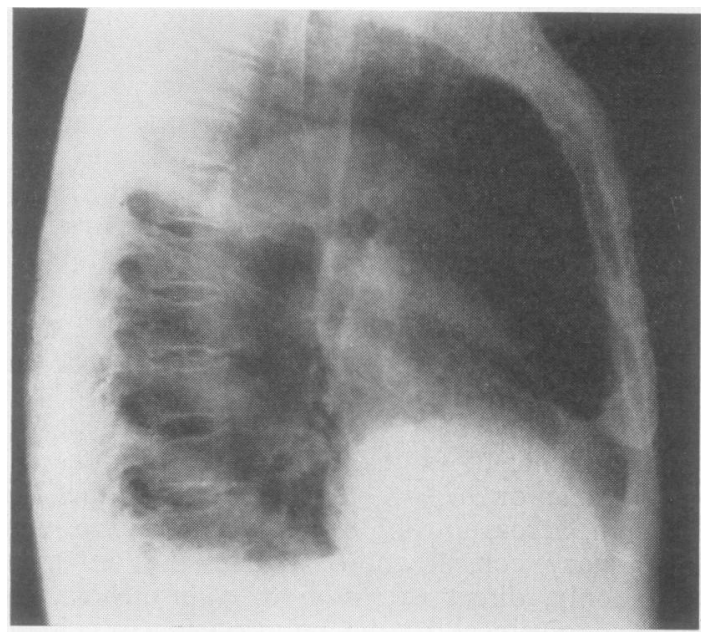

FIG. 10 coughing. There is, in these circumstances, an hepato-bronchial fistula. Repeated small haemoptyses may precede the expectoration of characteristic dark, reddish-brown sputum in which $E$. histolytica may be found. Radiographically, there is usually some evidence of a pulmonary lesion, and the diaphragm is elevated; a triangular shadow with its base on the diaphragm is said, in circumstances of known hepatic amoebiasis, to be characteristic. The prognosis is good. With appropriate treatment recovery is rapid in the patient with an hepato-bronchial fistula, but more protracted if there is a related pulmonary abscess. Complications such as empyema, metastatic abscess, and residual pulmonary fibrosis may occur. Surgical drainage is required only when the patient, to whom adequate medical treatment has been exhibited, deteriorates or fails to progress, and the decision to embark on a surgical procedure must not be made prematurely. Emetine, 
FIG. 11

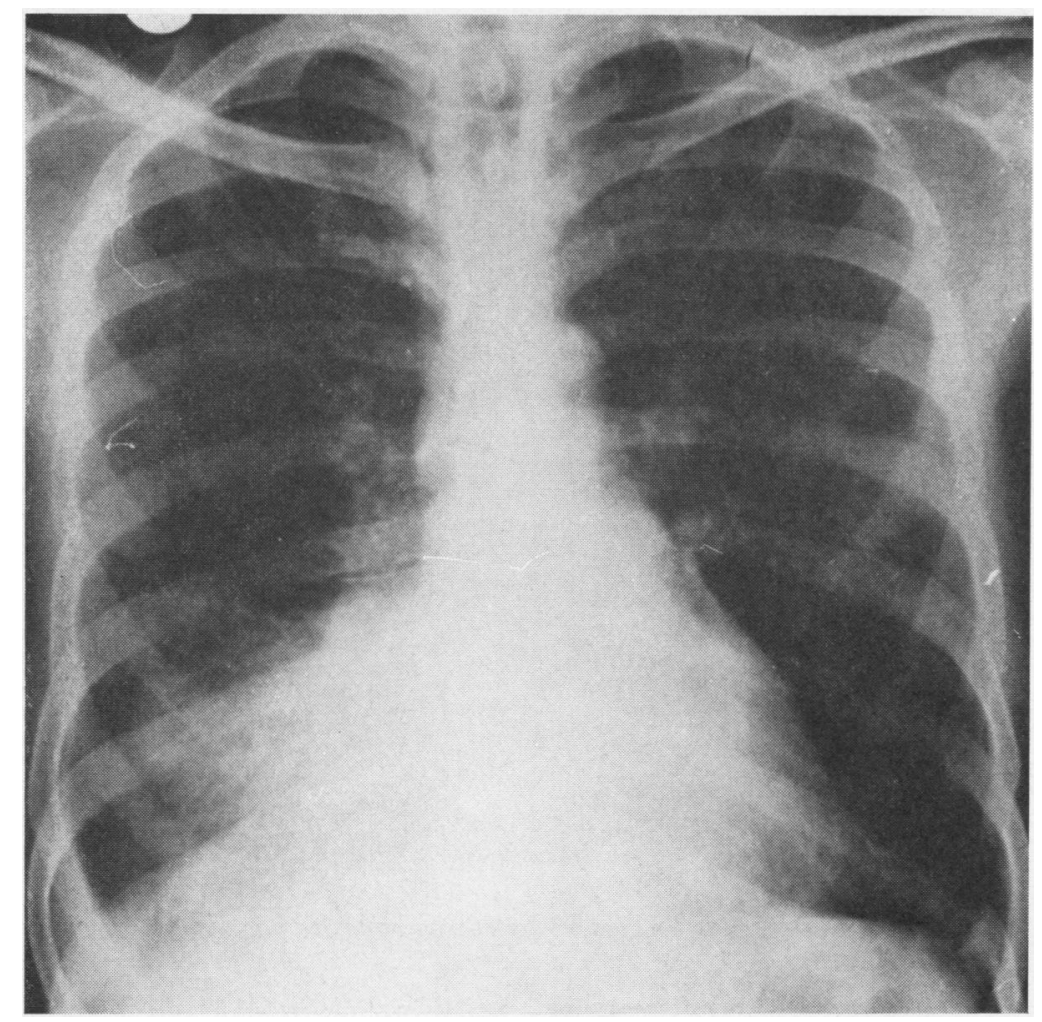

FIGS 11 and 12. There is a middle lobar opacity and a right pleural effusion. This patient was known to have hepatic amoebiasis. The pulmonary and pleural lesions cleared with appropriate drug therapy. E. histolytica was found in the sputum.

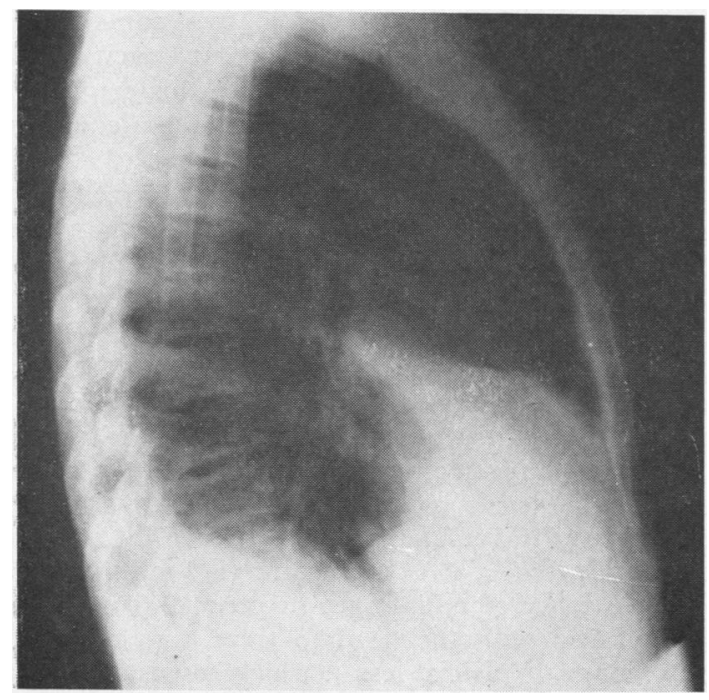

FIG. 12 chloroquine, and postural drainage will cure most amoebic lung abscesses; secondary bacterial contamination of the lung abscess may require appropriate chemotherapy. Resolution is the rule; occasionally, irreversibly damaged lung, with bronchiectasis or a chronic cavity, may require resection. Since an amoebic liver abscess is occasionally in communication with the biliary system, it sometimes happens that rupture of an abscess into the bronchus is followed by a broncho-biliary fistula which is recognized by the expectoration of bile-stained sputum; recovery with conservative management is again the rule.

In the community in the south-east region of Scotland, a persistent peripheral pulmonary opacity in the right lower lobe was so likely to be a carcinoma that, after an appropriate period of chemotherapy and after there had become available a sufficiently large series of radiographs to establish that the lesion was not clearing, management was usually by pulmonary resection. In 


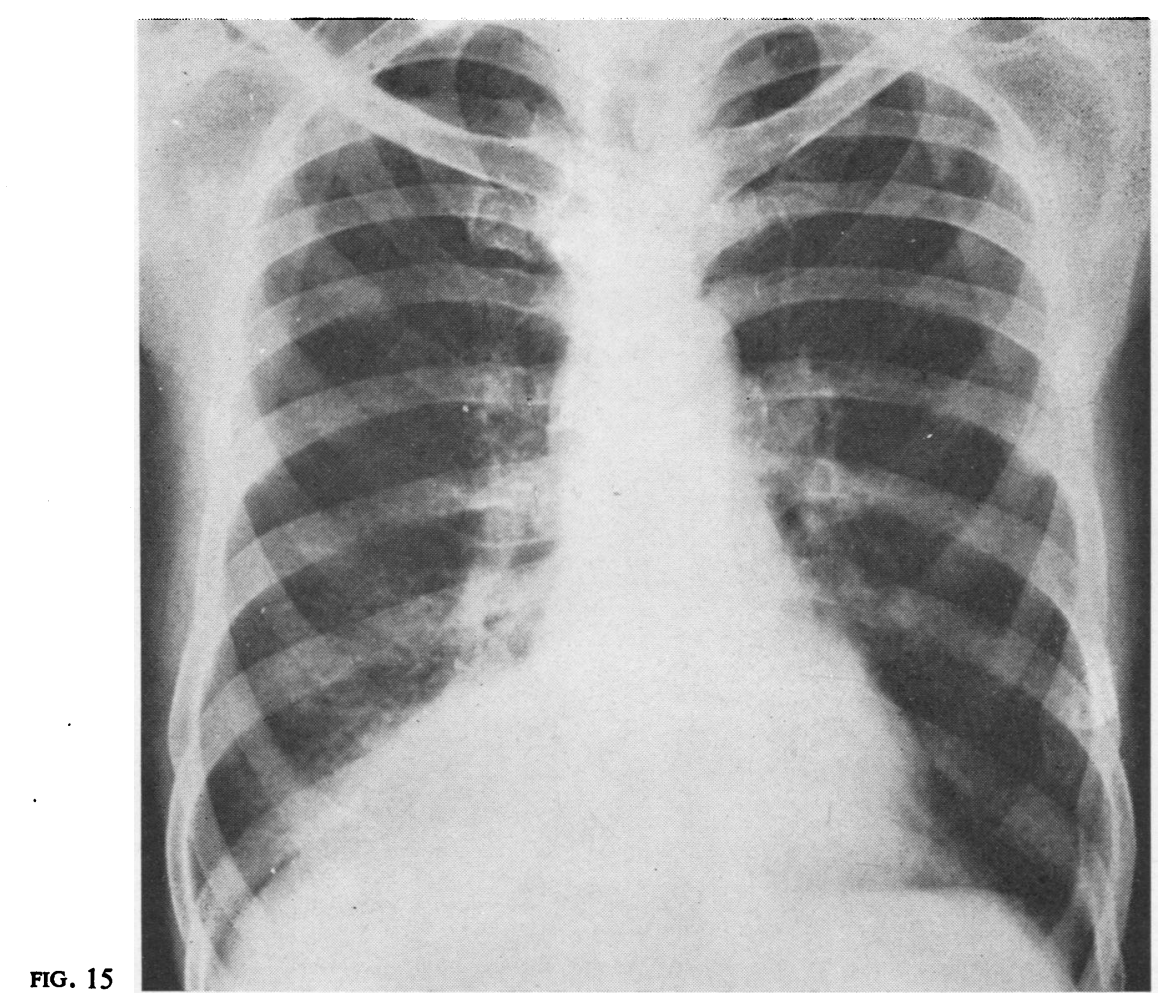

FIGS 13,14, 15, and 16. These radiographs are typical of the pulmonary and pleural changes which accompany hepatic amoebiasis which has extended through the diaphragm into pleura and lung. The appearances are nonspecific and the diagnosis of pleural and pulmonary amoebiasis is most likely to be achieved when the index of clinical suspicion is high, where it is known that amoebiasis is endemic or that there may have been exposure to infection elsewhere.

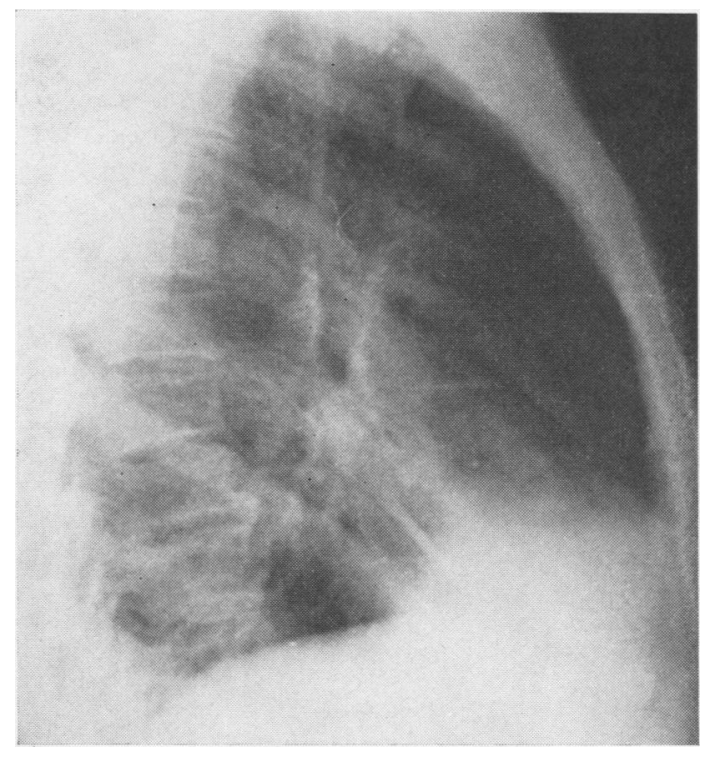

FIG. 16
There is both clinical and necropsy evidence of the occurrence of pulmonary amoebiasis in the absence of hepatic amoebiasis.

A lesion may develop in an area of the lung remote from the diaphragm, and this may reflect haematogenous spread, or transbronchial spread by aspiration or 'bronchial embolism', where there is a broncho-hepatic fistula. Many reports of pulmonary lesions attributed to amoebiasis are based on the association of a bowel infestation with $E$. histolytica, the disappearance of radiological opacities following the exhibition of emetine or chloroquine together with other therapy, or the demonstration of degenerate amoebae in the sputum. The validity of such accounts must be accepted with caution. Bowel infestation with $E$. histolytica is common in endemic areas and may co-exist with many other diseases; when infestation with $E$. histolytica co-exists with areas of pulmonary consolidation, the aetiology of which is never established, the apparent therapeutic effect of emetine may be no more than coincidental ; and particular 


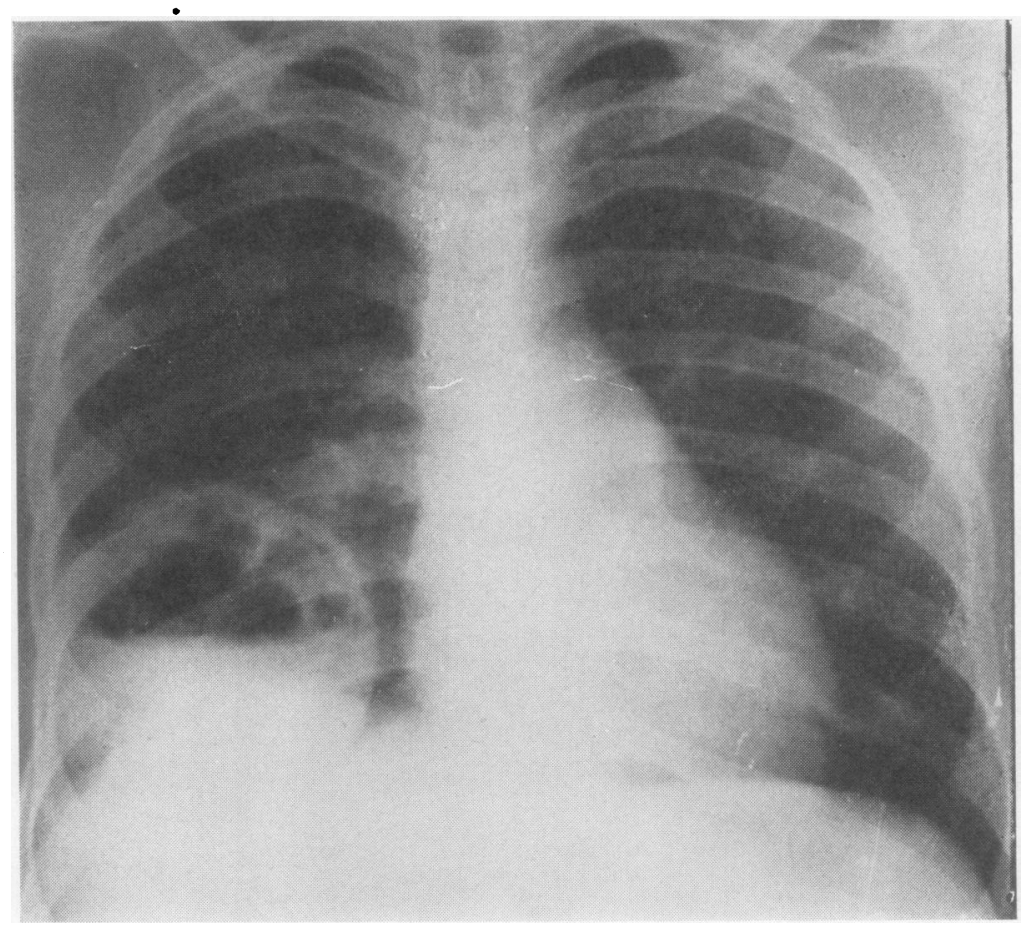

FIG. 17

FIGS 17 and 18. A right lower amoebic pulmonary abscess from which (over a 24-hour period) three pints of amoebic pus was evacuated by coughing. The interval between the films is four days. The appearance of the pus was typical and it was shown to contain $\mathrm{E}$. histolytica.

FIG. 18

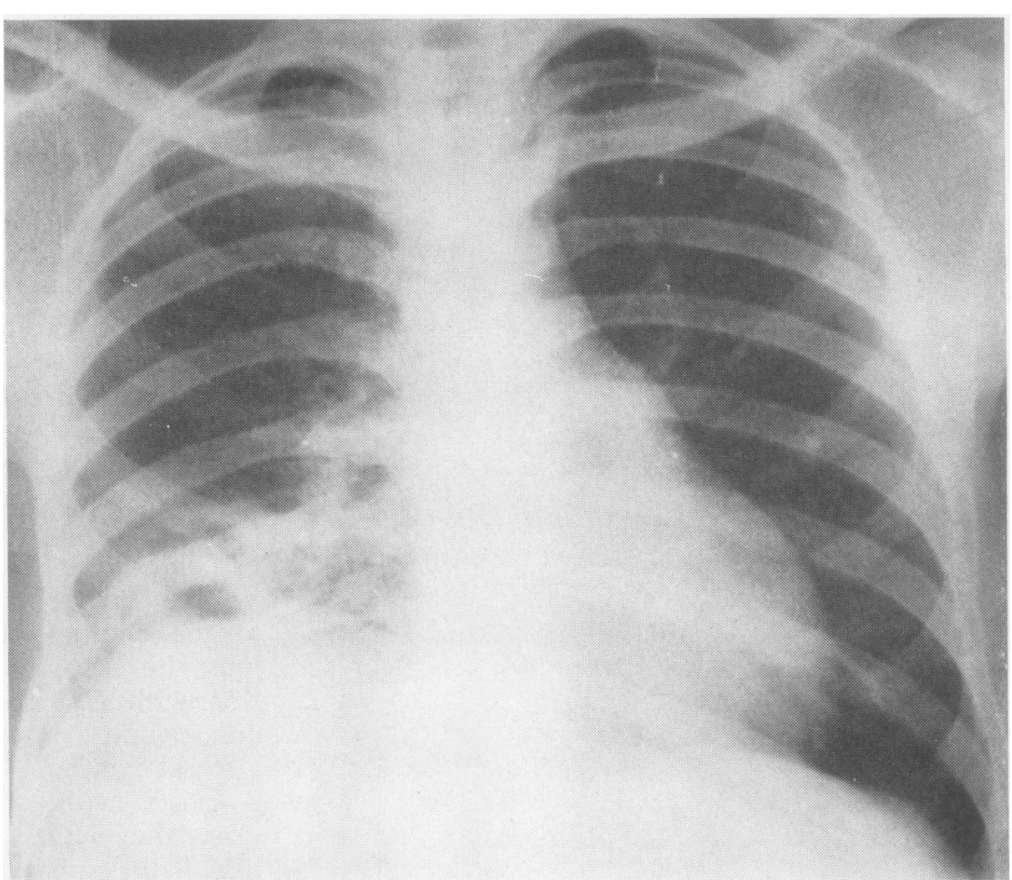


care is needed in the identification of amoebae in the sputum, since, unless morphological characteristics are unequivocal or the organism contains red blood cells, the parasite may easily be confused with Entamoeba gingivalis, a common oral commensal.

Single pulmonary lesions which may be haematogenous are more commonly described than are multiple lesions, and multiple lesions attributable to pulmonary amoebiasis are probably very uncommon.

Both bronchitis and asthma have been attributed to infestation with $E$. histolytica. It is assumed that the parasite reaches the bronchi from the gut by way of the bloodstream or, alternatively, its presence in the body constitutes an allergen. Evidence for such an association is unconvincing.

It is convenient to include here a brief discussion on amoebic pericarditis. In the majority of patients this complication is associated with an abscess in the left lobe of the liver (Macleod, Wilmot, and Powell, 1966). Right hepatic lobe lesions less often extend to the pericardium. Left empyema may complicate amoebic pericarditis rather than exist as a direct extension from the liver.

Amoebic pericarditis presents in two clinical forms: (1) as a pericardial rub with electrocardiographic and radiographic evidence of pericarditis associated with an amoebic liver abscess; or (2) as purulent pericarditis from perforation of an amoebic liver abscess into the pericardium.

In the first, prompt and successful treatment of the liver abscess is followed by resolution of the pericardial lesion. The second syndrome may have such an abrupt onset as to simulate one of the cardiac or pulmonary emergencies, and circulatory disturbances may quickly be followed by death. A less dramatic onset is more common. The diagnosis is confirmed by aspiration of pus from the pericardium; hepatic signs and the absence of bacteria from the pus should arouse suspicion that purulent pericarditis is in fact amoebic, particularly in an endemic area. The relief of cardiac tamponade is a matter of urgency; repeated aspiration may be necessary and open drainage is occasionally required; specific drug therapy is essential ; recovery without the development of constriction is usual. Where constriction has developed, secondary bacterial infection has sometimes been demonstrated and this may be the additional factor that determines the development of constriction. But constriction may develop in the absence of recognized secondary infection, and may resolve without recourse to pericardiectomy (Lamont and Pooler, 1958).

Pericardial aspiration is most safely undertaken through Larrey's angle or cleft (the angle between the ziphisternum and the left costal margin) on the left. The needle is introduced in a rostral and medial direction. Pericardial aspiration is more hazardous than aspiration of other serous cavities because of the danger of transgressing myocardium or puncturing a coronary vessel, with resultant haemopericardium or the induction of ventricular fibrillation. If the indifferent praecordial lead on the electrocardiograph is attached to a specially constructed electrode which connects the aspirating needle to the electrocardiograph machine and the electrocardiogram is monitored throughout the aspiration of the pericardial space, contact between the tip of the aspirating needle and epicardium will be seen to produce a characteristic cardiographic pattern, with ventricular premature systoles or an acute 'current of injury'. Although this technique does not make it impossible to damage a coronary vessel or the myocardium, it makes this very much less likely provided that reasonable care is taken. The use of a pericardial needle as an exploring electrocardiograph electrode to recognize signs of myocardial injury during pericardiocentesis has been fully recorded and has had a place in clinical practice established for some 10 years (Gotsman and Schrire, 1966).

\section{REFERENCES}

Gotsman, M. S., and Schrire, V. (1966). A pericardiocentesis electrode needle. Brit. Heart J., 28, 566.

Lamont, N. McE., and Pooler, N. R. (1958). Hepatic amoebiasis. A study of 250 cases. Quart. J. Med., $27,389$.

le Roux, B. T. (1965). Empyema thoracis. Brit. J. Surg., 52, 89.

Macleod, I. N., Wilmot, A. J., and Powell, S. J. (1966). Amoebic pericarditis. Quart. J. Med., 35, 293.

Maddison, S. E. (1965). Characterization of Entamoeba histolytica antigen-antibody reaction by gel diffusion. Exp. Parasit., 16, 224.

Wilmot, A. J. (1962). Clinical Amoebiasis. Blackwell, Oxford. 